\title{
Photomechanical Azobenzene Crystals
}

\author{
Takuya Taniguchi ${ }^{1}$ (D) Toru Asahi ${ }^{2,3}$ and Hideko Koshima ${ }^{3, *}$ \\ 1 Center for Data Science, Waseda University, 27 Waseda-cho, Shinjuku-ku, Tokyo 1620042, Japan \\ 2 Department of Advanced Science and Engineering, Graduate School of Advanced Science and Engineering, \\ Waseda University, 3-4-1 Okubo, Shinjuku-ku, Tokyo 1698555, Japan \\ 3 Research Organization for Nano \& Life Innovation, Waseda University, 513 Wasedatsurumaki-cho, \\ Shinjuku-ku, Tokyo 1620041, Japan \\ * Correspondence: h.koshima@kurenai.waseda.jp; Tel.: +81-3-5286-8307
}

Received: 12 August 2019; Accepted: 20 August 2019; Published: 22 August 2019

\begin{abstract}
Photomechanically responsive materials are promising candidates for future smart actuator applications. The photo-responsive behaviors originate from the photoisomerization of photochromic molecules. A typical photochromic compound, azobenzene, has been studied extensively in the solution state and has played a crucial role in the photomechanical behaviors of materials such as polymers and gels, via chemical bridging with their matrix. In contrast to polymers and gels, the photomechanical attributes of molecular crystals have not progressed to the same degree, due to their rigidity and fragility. However, the past decade has witnessed an increasing number of reports of the photomechanical motion of molecular crystals, including azobenzene crystals. This paper reviews the current state-of-the-art of mechanically responsive azobenzene crystals, including the history, crystal design strategy, and future promising applications.
\end{abstract}

Keywords: azobenzene; photomechanical motion; trans-cis photoisomerization; molecular crystal; actuation

\section{Introduction to Photomechanical Materials}

\subsection{Actuator Materials}

Actuation, which refers to mechanical motion or operation resulting from an energy conversion process, is prevalent throughout nature and characterizes some human activities. Animals and plants consume energy to create the mechanical motion required for survival and reproduction. Humans have developed mechanical systems for many purposes, and related to a wide range of actuation functions, to meet various needs. As to the specific materials used for actuation, inorganic materials that respond mechanically to external stimuli have historically played crucial roles in advancing actuation technology. For instance, a small piece of quartz, i.e., a crystal of silicon dioxide, ticks every second in an electric watch by oscillating at a constant frequency under an applied voltage. Shape memory alloys have been developed for many applications due to their shape recovery effect in response to a temperature change. Other examples of material actuation include those based on the piezoelectric effect, magnetostrictive effect, shape recovery, and thermal expansion [1].

The development of organic materials, mainly polymeric materials, for actuation has advanced steadily. Responses to various kinds of stimuli, including light, heat, voltage, magnetic fields, pressure, humidity, and biological enzymes have been achieved [2,3]. In addition, organic materials are generally softer, lighter, and cheaper than inorganic materials. Therefore, mechanically responsive organic materials are expected to come to the forefront in next-generation smart actuators. Potential future applications include soft robots, mechanical devices, active catheters, wearable applications, and so on $[4,5]$. 


\subsection{Azobenzene Compounds for Actuator Materials}

The photomechanical response upon light irradiation has advantages for remote control and diverse actuation via focused light or selective wavelengths [6]. Photomechanical polymers have been well developed by crosslinking the polymeric main chain with photochromic compounds, which convert their molecular geometry and electronic state upon photoirradiation [7]. The typical photochromic compound is azobenzene, which undergoes trans-to-cis photoisomerization and cis-to-trans back-isomerization reversibly (Scheme 1). The physicochemical properties of azobenzene compounds have been researched extensively in the solution state [8]. In polymeric networks, molecular geometry changes due to trans-to-cis photoisomerization result in photo-actuation. When a polymer crosslinked with azobenzene is irradiated by light at wavelengths suitable for photoisomerization, cis-isomer photoproducts are generated, mostly on the irradiated surface. The number of photoproducts decreases according to the penetration depth of the light. The gradient distribution of cis-isomer results in a bilayer-like structure in the material, inducing macroscopic deformations such as bending, twisting, and helicoidal motions [9-11]. This actuation mechanism can also be applied to azobenzene-containing gels. Gels are generally much softer than polymers due to the existence of solvents, and result in a photomechanical response with smaller output force $[12,13]$.

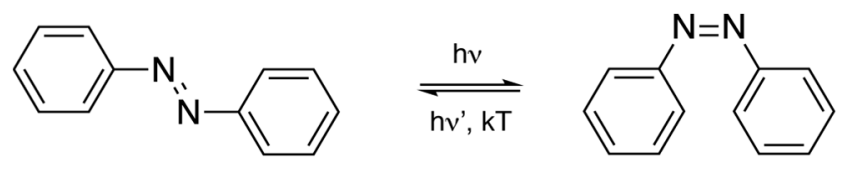

Scheme 1. Trans-to-cis photoisomerization of azobenzene upon photoirradiation (hv). Cis-to-trans back-isomerization undergoes by thermal energy $(\mathrm{kT})$ or light irradiation at different wavelengths $\left(\mathrm{h} v^{\prime}\right)$.

\subsection{Photomechanical Crystals}

In contrast to soft materials such as polymers and gels, molecular crystals are considered a more rigid and fragile material. For example, when strain is applied to a single crystal via photoproducts or mechanical stress, the periodically ordered alignment of molecules results in the collapse of a single crystalline state into a polycrystalline or amorphous state [14]. However, in contrast to this stereotypical characterization, a landmark report published in 2007 demonstrated that photochromic crystals of diarylethene compounds bent at an observable scale upon light irradiation [15]. This finding has had a significant impact on the research community. A decade later, interest in photomechanical crystals of various photochromic compounds continues to grow, as evidenced by the numerous reports in the literature [16-21].

Regarding typical photochromic compound azobenzenes, dozens of crystals have been reported to enable various photomechanical behaviors based on their trans-cis photoisomerization (Figure 1). Thus, azobenzenes have played a role in demonstrating the photo-responsive functionality of the crystalline state, prompting this review of its potential contribution to future developments in this research area.

Before moving to the next section, we briefly discuss the mechanical properties of mechanically responsive molecular crystals by comparing them to other actuator materials (Figure 2) [22]. Molecular crystals possess a Young's modulus of 1-10 GPa and deform with a strain of $0.1-1 \%$. Metals, inorganics, and shape memory alloys are located at the higher end of the Young's modulus range compared with molecular crystals. In contrast, polymers and gels reside in the lower modulus regime, however, their properties can be tuned over a broad range. The mapping of actuator materials shown in Figure 2 indicates that mechanically responsive molecular crystals are located in the unexplored area between hard and soft materials. This has motivated the search for new mechanical molecular crystals for novel and exciting applications. 

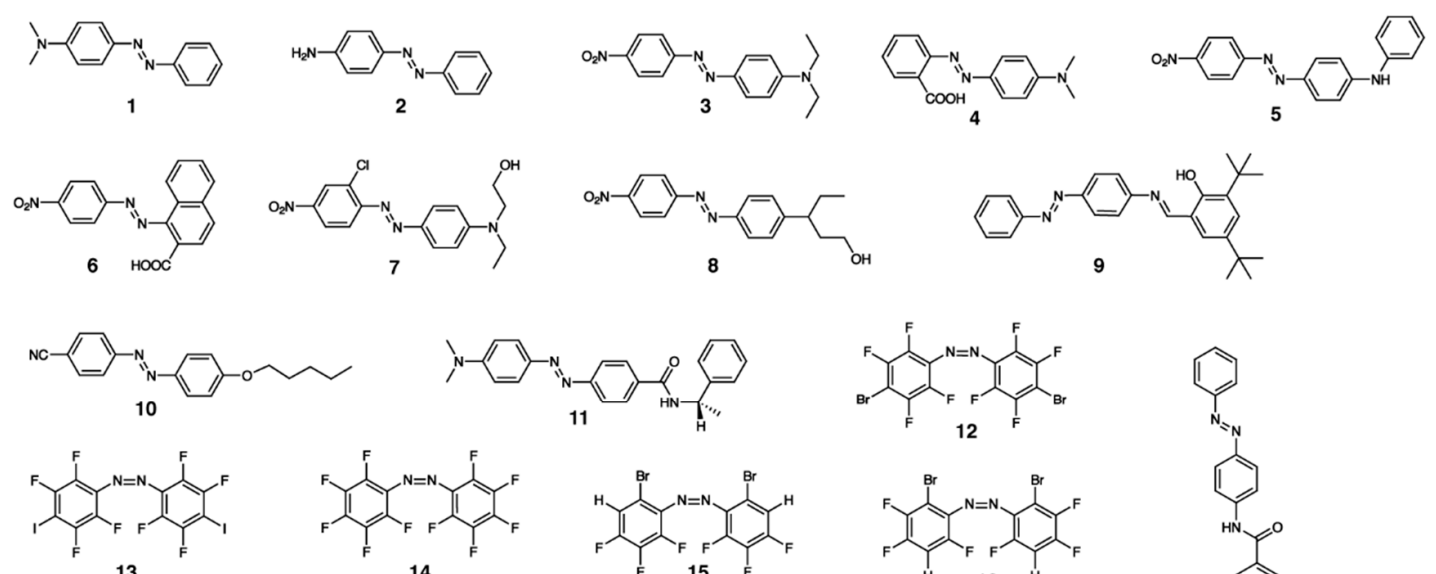

13
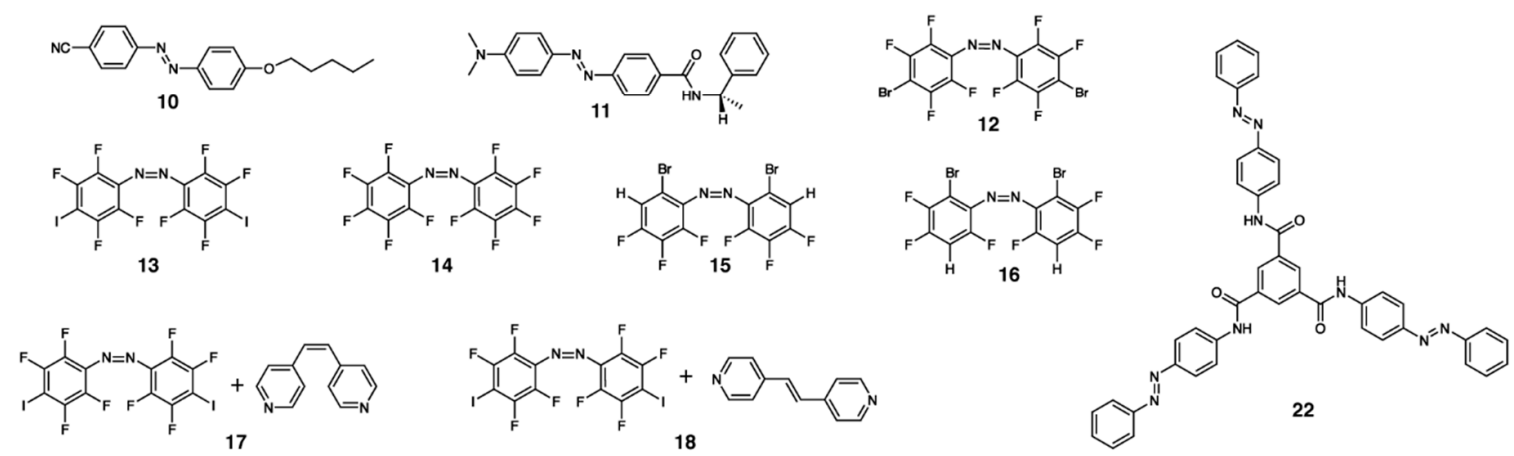<smiles>Fc1c(F)c(F)c(N=Nc2c(F)c(F)c(I)c(F)c2F)c(F)c1F</smiles>

19
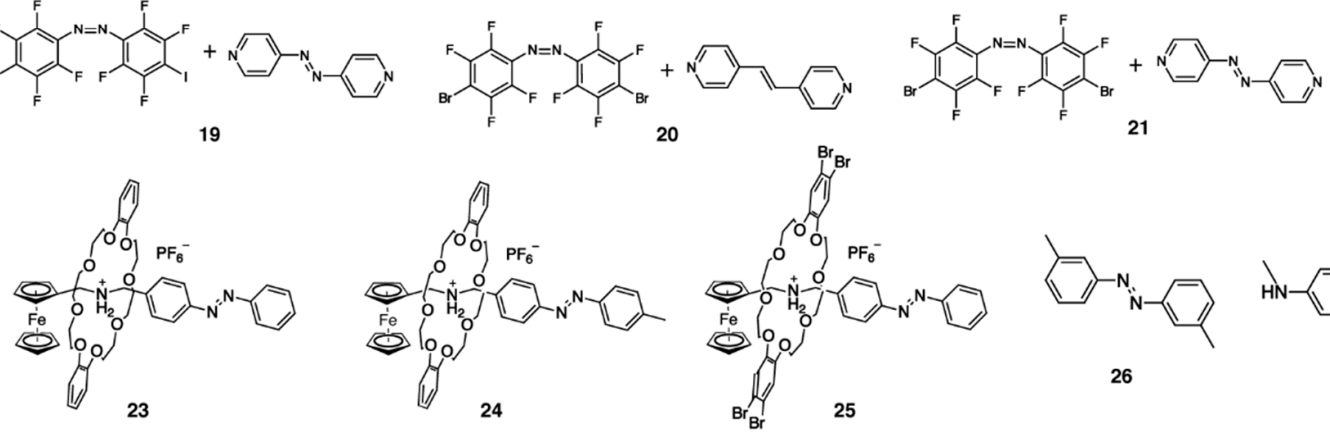

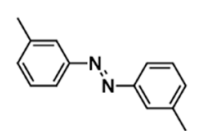

26

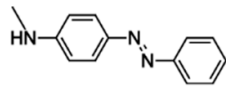

27

Figure 1. Photomechanical azobenzene crystals previously reported in [26] for 1, [27] for 2, [29] for 3-7, [32] for 8, [33] for 9, [49] for 10, [38] for 11, [42,43] for 12-16, [44] for 17-21, [53] for 22, [45] for 23-25, [46] for 26, and [47] for 27.

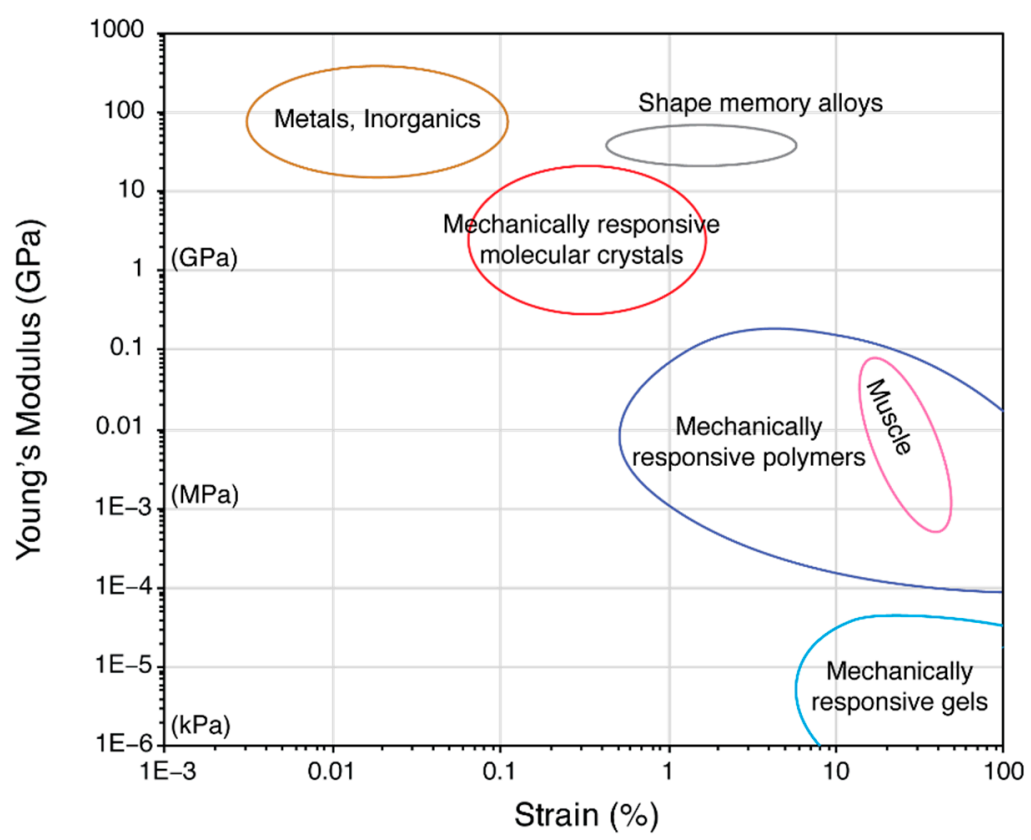

Figure 2. Relationship between the Young's modulus and strain of actuator materials. Reprinted with permission from [22]. Copyright 2019 Wiley-VCH. 


\section{Development of Photomechanical Azobenzene Crystals}

\subsection{Early Stage of Photomechanical Azobenzene Crystals}

Although the crystal structures of trans- and cis-azobenzene with no substituent were determined almost 50 years ago $[23,24]$, trans-cis photoisomerization in the crystalline state had been thought not to occur at all [25], or to occur only under controlled circumstances, as the molecular transformation requires motion of molecules in a densely packed space. However, reports on the photomechanical bending of diarylethene crystals has motivated renewed exploration of photomechanically bendable azobenzene crystals.

A crystal of trans-4-(dimethylamino)azobenzene (trans-1) was reported as the first photomechanical azobenzene crystal [26]. A thin plate-like crystal of trans-1, which was prepared by sublimation, bent along the $b$-axis upon ultraviolet (UV) light irradiation onto the (001) surface (Figure 3 ). The bending reached a steady state in $5 \mathrm{~s}$ under light irradiation, and then returned to the initial unbent shape within $30 \mathrm{~s}$ upon the removal of UV irradiation. Notably, reversible bending was demonstrated over 100 cycles [26].

The bending response originates from the strain induced by trans-to-cis photoisomerization via a mechanism similar to that found in polymers. The photoisomerization is most significant at the irradiated surface and decreases depending on the light penetration depth. The gradient formation of cis-isomers generates strain in the thin crystal, resulting in a bending motion away from the light source. One of the critical factors related to the bending response is the electron donor substituent of $\mathbf{1}$. The amino group works as an electron donor and increases the photoreactivity of the $\mathrm{n}-\pi^{*}$ transition, through which trans-to-cis photoisomerization occurs [8]. The increased reactivity allows conversion of trans-1 into cis-1 in the crystal, which is sufficient for bending by the generated strain.

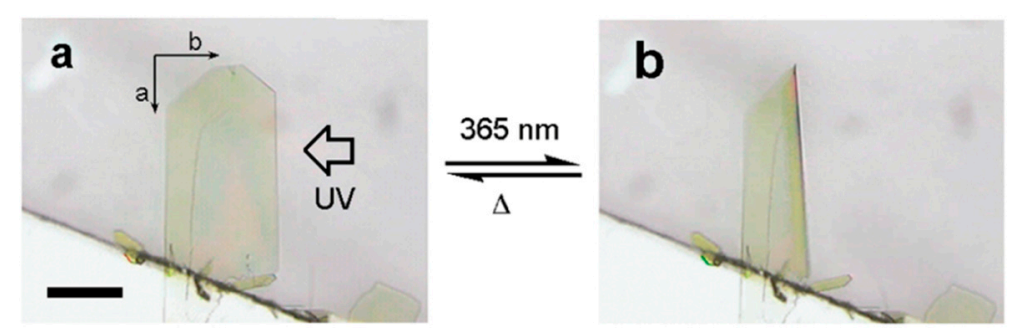

Figure 3. Photomechanical bending of a thin crystal of trans-1 (a) before and (b) after ultraviolet (UV) irradiation. The irradiation was conducted from the right rear side of the crystal, as indicated by the arrow. The scale bar is $200 \mu \mathrm{m}$. Reprinted with permission from [26]. Copyright 2009 American Chemical Society.

Shortly after the initial report, trans-4-aminoazobenzene (trans-2) bending under UV irradiation was demonstrated [27]. As to the molecular structure, trans-2 has also been used as an electron donor substituent. A thin crystal of trans-2, whose longitudinal direction corresponds to the crystallographic $b$-axis, bent away from the light source upon UV irradiation from the left side (Figure 4). After turning off the light, the bending returned to the initial state within $240 \mathrm{~s}$. However, when irradiated by visible light $(530 \mathrm{~nm})$ the initial state was achieved within $60 \mathrm{~s}$. These findings suggest that tuning the photoreactivity of azobenzene compounds provides the means to construct photomechanical azobenzene crystals. 

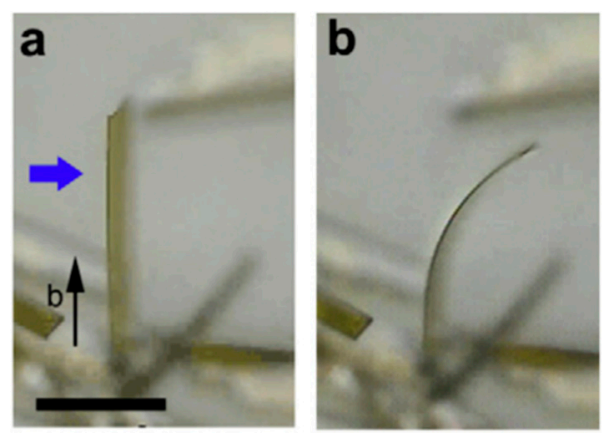

Figure 4. Photomechanical bending of a thin crystal of trans-2 (a) before and (b) after UV irradiation. The scale bar is $100 \mu \mathrm{m}$. Reprinted with permission from [27]. Copyright 2012 Elsevier.

\subsection{Fast Response}

Photomechanical crystals show dynamic photo-responsive deformations, however, the bent crystal takes tens of seconds or minutes to return to its initial shape, limiting practical application. The reversible bending can be divided into two processes: a photomechanical bending process due to trans-to-cis photoisomerization and an unbending process due to cis-to-trans thermal or photochemical back-isomerization. Thus, the bending process depends on the light conditions (e.g., intensity and wavelength) and the photoreactivity (for trans-cis photoisomerization). The consecutive unbending process is affected by the half-life of cis-trans back isomerization. Of course, both processes also depend on the size and shape of crystals and other external conditions, such as temperature.

The fast relaxation of cis-to-trans back isomerization can be tuned by adding an electronwithdrawing group to an azobenzene [28]. The electron-withdrawing group works to pull the electrons, resulting in the short lifetimes of cis-isomers relative to other azobenzenes. The electron-withdrawing group also shifts its absorption peak into wavelengths ranging from blue to green, enabling a response to visible light. Bushuyev et al. reported fast reversible bending of azobenzene crystals $(1,3-7$ in trans-form) by irradiation with high-power visible light [29]. Among them, thin crystals of 3-7, which have an electron-withdrawing $-\mathrm{NO}_{2}$ or $-\mathrm{COOH}$ group, exhibited a faster unbending speed at half-lives of less than $0.1 \mathrm{~s}$ after the removal of high-power light irradiation of $1 \mathrm{~W} / \mathrm{cm}^{2}$. The crystals of 3-7 in trans-form required minimal light intensities of $50-200 \mathrm{~mW} / \mathrm{cm}^{2}$ for photo-bending, whereas the trans-1 crystal initiated bending at $1 \mathrm{~mW} / \mathrm{cm}^{2}$ due to its higher photoreactivity.

\subsection{Model of Dynamical Photo-Bending}

There have been an increasing number of reports of photomechanical azobenzene crystals, however, a quantitative mathematical description of the mechanical response has not yet been provided. The classical bending model is based on the bimetal model described by Timoshenko [30]. When a plate with a specific coefficient of thermal expansion is attached to another plate that has a different coefficient of thermal expansion, the bilayer material bends upon heating towards the material with the lower coefficient due to the generated strain. In the case of photomechanical actuation, the bending strain originates from photoproducts, which form nonuniformly in the crystal. The structural mismatch between the photoproduct and the reactants creates a residual strain that causes macroscopic deformation of the crystal. Although Timoshenko's bimetal model can explain the bending speed of photomechanical crystals [31], it is essential to establish a mathematical model that provides a kinematic explanation of the macroscopic reshaping.

Naumov et al. constructed two kinematic models, one simple model and one extended model, to describe the bending angle as a function of time based on reaction kinetics theory [32]. The simple model considers the rate constants of the primary reactions between the reactant and photoproduct and describes bending and relaxation as monolithic exponential models. The extended model is a biexponential model that considers chemical processes in detail, including the excited state, intersystem crossing, and internal conversion. The two models were verified by reference to the experimental 
results of slender azobenzene crystals of trans-8 (Figure 5). The time profile of the bending angle during photomechanical bending was fully fitted to the simple model, and was also well explained by the extended model. The bending angle during the relaxation process was not well explained by the simple model, but well fitted the extended biexponential model. Such kinematic model was further extended as described in their review [19]. They also reported photomechanical behavior of 9 [33].

a

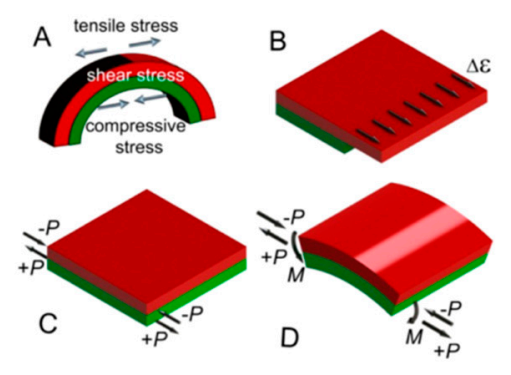

b

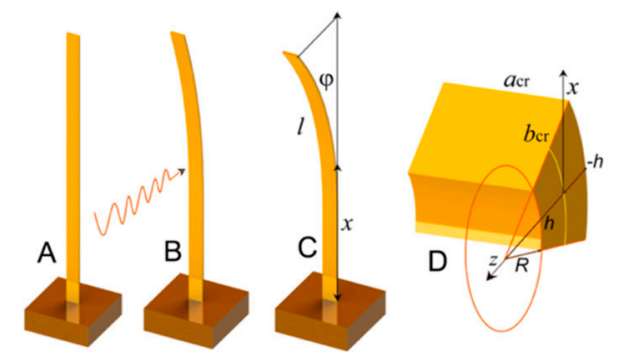

C
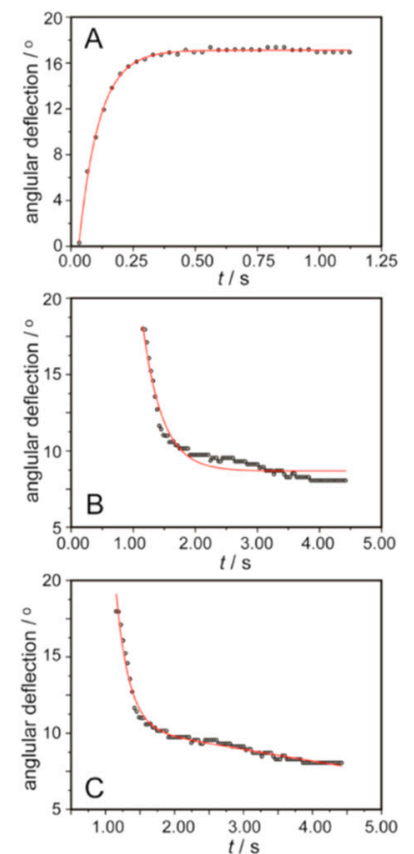

Figure 5. Model construction of the kinematic behavior of photo-bending. (a) Bilayer model of bending. (b) Definition of angular deflection and related parameters. (c) Model verification by reference to the experimental results of slender photomechanical crystals of trans-8. The models include a simple model for bending (A), a simple model for unbending (B), and an extended model for unbending (C). Reprinted with permission from [32]. Copyright 2014 American Chemical Society.

\section{Design of New Azobenzene Crystals for Actuation}

Molecular crystals provide a platform for various functionalities, as organic molecules can form diverse structures with limited constituents. The strategy used to functionalize a crystal relies on the crystal design, specifically, the molecular structure and intermolecular interactions. The molecular architecture provides the basis for new crystal structures expressing specific functions, although it is challenging to predict crystal structures from molecular structures. This section reviews several works describing photomechanical azobenzene crystal construction, according to the crystal design.

\subsection{Chirality Induction}

Dozens of photomechanical crystals, not of only azobenzenes but also of other photochromic compounds, have been reported to bend under photoirradiation. The bending motion is a mode of actuation resulting from elongation or contraction of the irradiated surface. In contrast to bending, there have been few reports on twisting of the crystal, which is another mode of actuation [34-37]. The twisted shape has chirality, because the right-handed twist does not overlay its mirror image, i.e., the left-handed twist. Thus, photoisomerization in chiral photochromic crystals may lead to macroscopic deformation with chirality, such as a twisting motion.

Based on this concept, the photomechanical motion of thin crystals of compound trans-(S)-11 was revealed [38]. In this study, a thin plate crystal was obtained by sublimation. The crystal had a (001) face with the $b$-axis as the longitudinal axis, along which intermolecular $\mathrm{NH}$---O hydrogen bonding 
chains formed in the crystal. When the front (001) face was irradiated with UV light, the crystal bent away from the source with slight left-handed twisting (Figure 6). The crystals, which showed bending with the slight twist, returned to their initial shape within 2-3 min after turning off the UV light. When the back (00-1) surface was irradiated, bending motion with a twist was observed, similar to that of the (001) face (Figure 6).

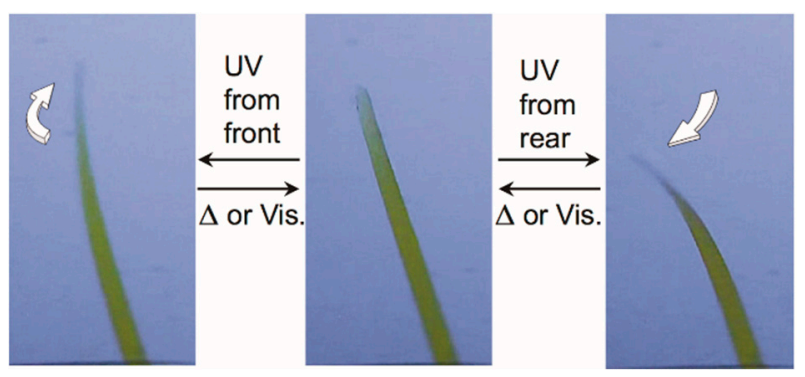

Figure 6. Photomechanical reversible bending with twisting of a chiral azobenzene crystal of trans-(S)-11. Upon UV irradiation on the front (001) face, the thin crystal bends with a left-handed twist. Bending in the same direction as the twist was seen upon light irradiation on the back (00-1) face. Reprinted with permission from [38]. Copyright 2016 Wiley-VCH.

This bending accompanying a twist is caused by elongation of the irradiated surface along the orthogonal direction. At the molecular level, trans-to-cis photoisomerization of compound trans-(S)-11 leads to contraction of the $a$-axis and elongation of the $b$-axis, based on the optimized molecular geometries of cis-(S)-11. This change in molecular structure occurs mostly on the irradiated surface, and the probability of trans-to-cis photoisomerization decreases along the thickness direction due to the attenuation of UV light. This is why cis-(S)-11 molecules are formed in the crystal along a gradient from the irradiated surface. The strain induces elongation along the $b$-axis and contraction along the $a$-axis, resulting in bending away from the light source with a twist.

\subsection{Crystallization with Multi-Molecular Components}

Another strategy is to design photomechanical azobenzene crystals with multi-components. One notable crystal engineering technique is co-crystallization. A co-crystal is defined as a crystal consisting of at least two molecules. Co-crystals can be obtained by tuning intermolecular interactions, for instance hydrogen bond and halogen bond interactions. To induce intermolecular interactions between two components, it is of key importance to design one component as a donor and the other as an acceptor of the expected intermolecular interaction. To date, there have been numerous reports on co-crystallization techniques, and the functions of co-crystals, where the number of combinations of two components is nearly infinite and affords diverse crystal structures [39-41].

The crystals of fluorinated azobenzenes 12-16 in cis-form exhibit photomechanical bending due to cis-to-trans photoisomerization upon visible light irradiation [42,43]. Photomechanical azobenzene co-crystals 17-21 were developed by constructing halogen bonds between halogenated azobenzene as a donor and a pyridine compound as an accepter [44]. The halogen I- or Br- at the para-position of the electron-deficient perfluorphenyl group is highly polarizable and works as a donor, forming the linear interaction between an accepter of the halogen bond, pyridine. The halogen bonding motif results in a zig-zag crystal structure, with alternating alignment of the donor and acceptor molecules, for example at a pitch of $20 \AA$ in the case of the co-crystal of 17 (Figure 7). Here, the co-crystal consists of cis-azobenzene, despite the lower stability of the cis-isomer compared with the trans-isomer; the cis-trans thermal back-isomerization of fluorinated azobenzene is very slow, allowing retention of the cis-isomer during recrystallization. 


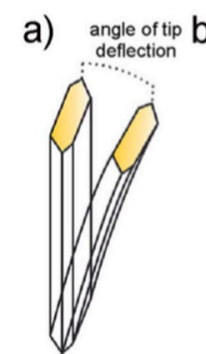

c)

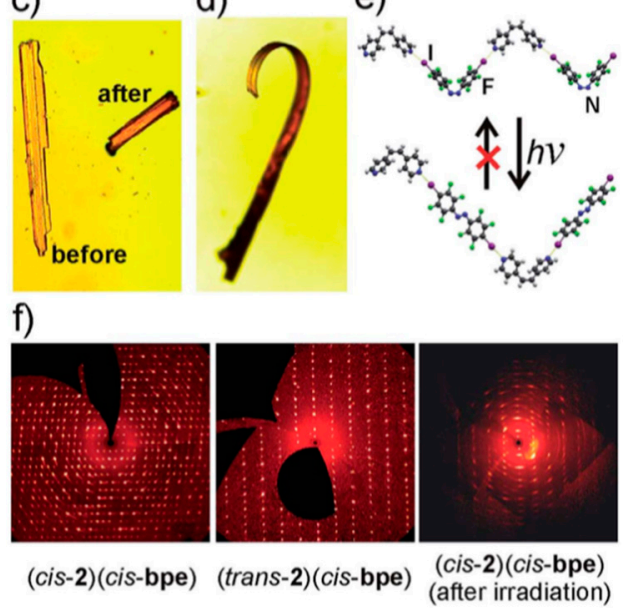

Figure 7. Irreversible photomechanical bending of a plate-like co-crystal of 17. Reprinted with permission from [44]. Copyright 2014 Royal Society of Chemistry.

All co-crystals of 17-21 exhibit photomechanical bending under 532-nm laser irradiation, however, the deflection angle depends on the components and partners of the co-crystals. The most bendable co-crystal under weaker light intensity $\left(5 \mathrm{~mW} / \mathrm{cm}^{2}\right)$ was that of $\mathbf{1 7}$ (Figure 7). Upon light irradiation, the crystal bent away from the light source up to a deflection angle of $90^{\circ}$. The bent crystal did not return to its initial shape after the removal of irradiation, indicating irreversible photomechanical bending due to cis-to-trans photoisomerization upon visible light irradiation. When a cis-isomer transforms to a trans-isomer, the trans-isomer forms a halogen bond with another pyridine, leading to a new co-crystal structure made from the trans-isomer. The structural mismatch between the new daughter crystal and the mother co-crystal of the cis-isomer produces the strain to bend away. The presence of a small amount of co-crystals in the trans-isomer was confirmed experimentally via in-situ X-ray crystallographic analysis, which also revealed that the conversion process unfolded as a crystal-to-crystal process (Figure 7).

Besides co-crystals, molecular machines such as rotaxanes are also composed of multi-components with an axial molecule and a ring molecule. Photo-reactive pseudorotaxane crystals of 23-25 were constructed with an axial element with azobenzene and a ring element with different substituents [45]. The pseudorotaxanes with different substituents afforded crystal structures with differing intra- and intermolecular $\pi-\pi$ distances and angles. All crystals of $23-25$ bent upon UV ( $360 \mathrm{~nm}$ ) and/or visible light $(445 \mathrm{~nm})$ irradiation due to trans-cis photoisomerization, although the bending angle varied with the crystal structure.

\section{Other Mechanical Responses of Azobenzene Crystals}

Besides bending and twisting behaviors, some azobenzene crystals reportedly exhibit movement, that is, locomotion, on a substrate surface upon encountering an external stimulus. Locomotion of a material from one position to another is potentially useful for the transfer of compounds, and to penetrate small spaces. Effectively, locomotive molecular crystals may work as small robots in some environments. Two locomotive features of azobenzene crystals are described in the following. 
First, crawling locomotion occurs due to a change in the physicochemical properties of the crystal caused by trans-cis photoisomerization [46]. When rhombus-shaped crystals of 3,3'-dimethylazobenzene (26) were irradiated by UV light from one direction, and simultaneously by visible light from the opposite direction, the crystals moved, i.e., crawled, very slowly on the glass surface in the direction of visible light irradiation (Figure 8). Phenomenologically, crawling locomotion of the crystal originates from the melting and crystallization of opposite surfaces via light irradiation. The crystals of $\mathbf{2 6}$, whose melting temperature is $c a$. $51-54{ }^{\circ} \mathrm{C}$, melt upon UV irradiation at room temperature, due to the depression of the melting point induced by trans-to-cis photoisomerization. On the back side, visible light irradiation causes cis-to-trans photochemical back-isomerization, resulting in recrystallization of trans-26. This suggests that the retraction caused by UV-light-induced melting, and crystal growth caused by visible-light-induced recrystallization, are likely responsible for crawl locomotion on the glass surface. Recently, the crawling motion of crystals of $\mathbf{2 7}$ was achieved by using only visible light [47]. Such solid-to-liquid transitions induced by photoisomerization also enable swimming on water surfaces [48] and multi-directional bending of thin crystals of compound 10 [49].

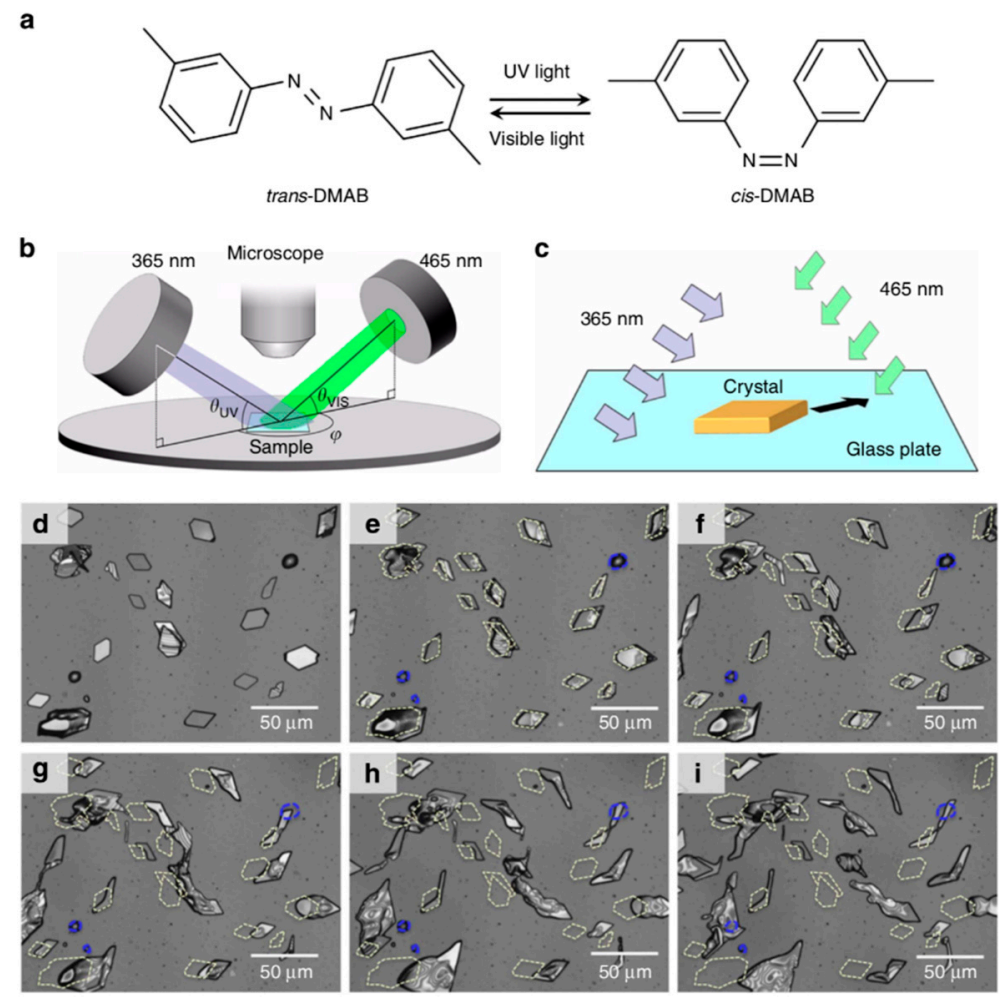

Figure 8. Light-induced crawling of crystals of 3,3'-dimethylaminoazobenzne (26). Reprinted with permission from [46]. Copyright 2015 Springer Nature.

Second, thermal locomotion is induced by structural phase transitions in trans-(S)-11 crystals, which is also expressed as photomechanical motion, as described previously [50]. In this study, the thermal structural phase transition was unexpectedly found to be a reversible process at $145^{\circ} \mathrm{C}$ upon heating and cooling. This phase transition proceeds in a single-crystal-to-single-crystal manner, without collapse of the single crystal. Due to the phase transition, the length of the $b$-axis, the longitudinal direction of a plate-like crystal, decreases by $0.3 \%$ at temperatures higher than $145{ }^{\circ} \mathrm{C}$, and returns to the initial length at lower temperatures. Here, an azobenzene molecule in a unit cell changes its conformation slightly but maintains trans-form during the phase transition. The decrease of, and return to the original, crystal length lead to bending motion at the phase transition, due to the temperature gradient along the thickness direction. Upon heating, the phase transition starts at the lower side of the low-temperature (LT) phase crystal, and the crystal bends due to shortening of the crystal length. With 
additional heating, the whole crystal completes the phase transition to a high-temperature (HT) phase. The HT phase crystal returns to the LT phase upon cooling through additional bending motion, as the transition to the LT phase occurs at the upper side cooled by the surrounding air. In turn, this leads to elongation of the crystal at the upper side. Thus, the crystal exhibits bending during both the heating and cooling cycles.

When a plate-like crystal with thickness gradient along the length was repeatedly heated and cooled near the phase transition temperature on a silanized glass, it was noted that the crystal "walked" in the manner of an inchworm due to repeated bending and straightening (Figure 9). In another case, a thin plate-like crystal moved much faster by rolling on a glass surface after a single heating or cooling process. In the cases of both walking and rolling, the locomotion is induced by the asymmetric shape of the crystal. Walking occurs according to the thickness gradient, where one side is thicker than the other, leading to unidirectional movement via bending and straightening. Rolling occurs according to the width gradient, and leads to loss of balance during bending and then flipping.

a

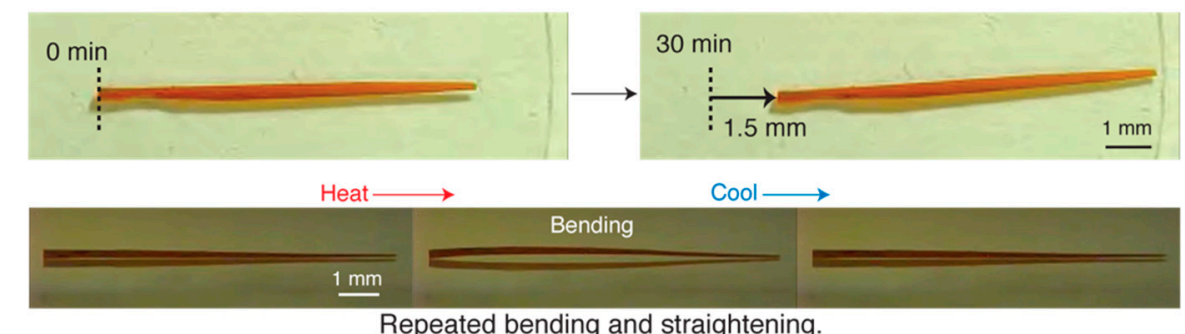

Repeated bending and straightening.

b

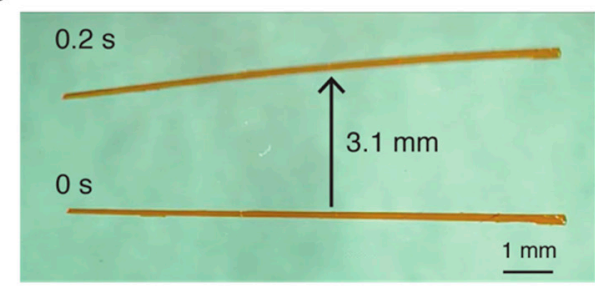

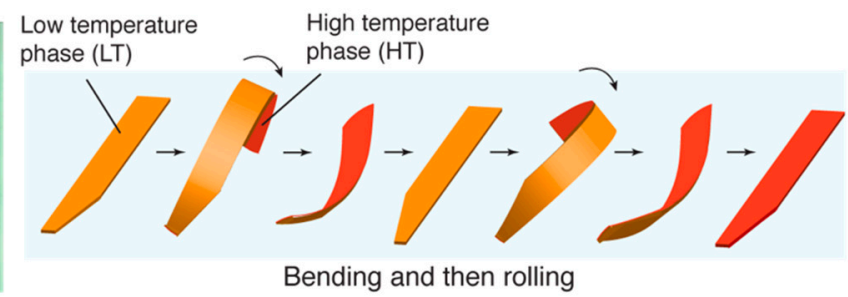

Figure 9. Thermal locomotion of crystals of trans-(S)-11 due to the structural phase transition. (a) Inchworm-like "walking" of a crystal with a thickness gradient along the length under cycles of heating and cooling. (b) Rolling of a thin crystal with a width gradient upon heating or cooling. Reprinted with permission from [50]. Copyright 2018 Springer Nature.

These examples suggest that the mechanical responses of molecular crystals can be diversified by a photoisomerization-induced phase change from the crystal form to a melted form, and by thermal structural phase transitions. The mechanical function of the structural phase transition can be combined with the photomechanical response of photochromic crystals. Combining the structural phase transition with photoisomerization has resulted in multiple mechanical motions of crystals [51,52].

\section{Possibilities for Future Applications}

So far, we have reviewed the mechanical responses of azobenzene crystals. The actuation mechanism is interesting from a purely scientific standpoint. However, it is important to remember that mechanically responsive crystals also show great potential as smart actuators and will find appropriate applications as the field advances.

A straightforward example of implementation is provided by photomechanical bending of azobenzene crystals, applied to the gripper of a micropipette (Figure 10) [53]. Here, the micropipette was constructed using the arm of an azobenzene compound 22 nanowire on the left side, and an additional fixed polystyrene nanowire arm on the right side. When the pipette arms were irradiated by UV light, the azobenzene nanowire bent towards the light source, owing to the transparency of 
polystyrene. The bending of the azobenzene nanowire led to the grabbing of a small particle. This is one of many possible applications of azobenzene crystals while other potential applications include microelectromechanical systems (MEMS), soft robots, flexible devices, medical catheters, aerospace devices, and so on. Recent advances in 3D and 4D printing technology could be further improved by mechanically responsive molecular crystals, owing to inherently advantageous properties such as light weight and softness $[54,55]$.

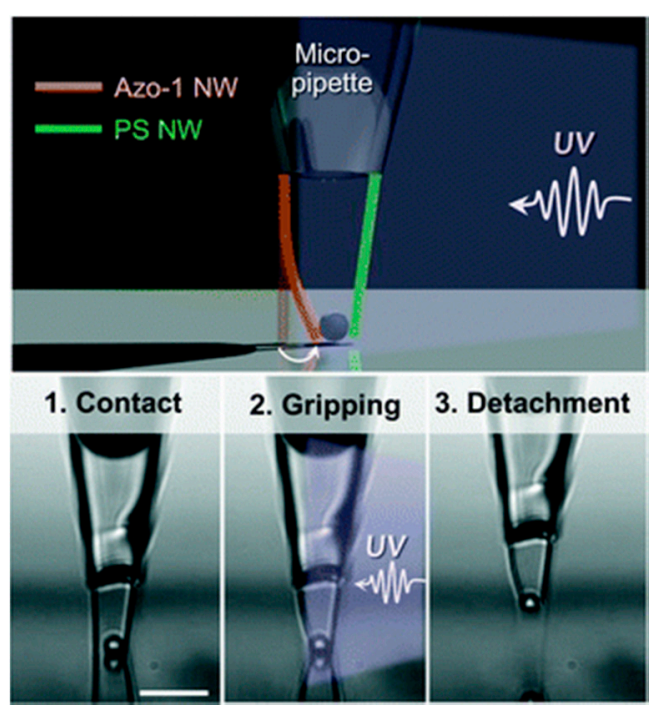

Figure 10. Demonstration of a micro-pipette using azobenzene nanowire of compound 22. Azobenzene nanowire bends towards the light source and grips a particle; the polystyrene (PS) nanowire is a transparent supporter. Reprinted with permission from [53]. Copyright 2015 Royal Society of Chemistry.

However, there are still some problems limiting the application of azobenzene crystals. The first concerns the difficulty of increasing the maximum force at actuation, and the second concerns the difficulty of fabricating the desired shape. Regarding the first one, mechanically responsive molecular crystals typically generate a maximum stress in the range of $1-50 \mathrm{MPa}$ at actuation $[50,56,57]$. These values are generally 10-100 times larger than the maximum stress of typical human muscle (0.3 MPa) [58]. To lead larger stress to larger output force, multiple pieces of crystals need to be integrated. Also, it is challenging to fabricate molecular crystals with the desired shape and size.

The hybridization of crystals and polymers is a promising approach to overcome the limitations discussed above [59]. When molecular crystals are incorporated into a connective polymer, the hybrid material will be more flexible and easier to control, in terms of size and shape, due to the properties of the polymer. In addition, such hybrid materials should respond faster and generate a larger force than polymers, owing to the inherent advantages of molecular crystals. Thus, the hybrid strategy of combining a polymer with a mechanical crystal, expected to be realized in the near future, should enhance the maximum force capability and simplify the fabrication process of actuator materials.

\section{Conclusions}

This paper reviewed the current state-of-the-art of photomechanical azobenzene crystals. Photomechanical responses result from simple trans-cis photoisomerization, but lead to a wide array of photomechanical behaviors varying by actuation mode (bending or twisting), bending magnitude, response speed, and relaxation time. Tuning azobenzene substituents and employing crystal engineering techniques, such as co-crystallization, have crucial roles in diversifying the photo-responsiveness and functionality of azobenzene crystals. Although there are still challenges ahead, photomechanical crystals show promise as novel actuators, with possible applications in soft robots, and medical and flexible devices. 
Author Contributions: Writing—original draft preparation, T.T.; writing—review and editing, T.T. and H.K.; supervision, T.A. and H.K.

Funding: This research was funded by the JSPS Grant-in-Aid for Scientific Research B (17H03107) and Challenging Exploratory Research (16K12918).

Acknowledgments: T.T. and T.A. thank the Leading Graduate Program in Science and Engineering at Waseda University.

Conflicts of Interest: The authors declare no conflict of interest.

\section{References}

1. Liu, K.; Cheng, C.; Cheng, Z.; Wang, K.; Ramesh, R.; Wu, J. Giant-amplitude, high-work density microactuators with phase transition activated nanolayer bimorphs. Nano Lett. 2012, 12, 6302-6308. [CrossRef] [PubMed]

2. Montero De Espinosa, L.; Meesorn, W.; Moatsou, D.; Weder, C. Bioinspired polymer systems with stimuli-responsive mechanical properties. Chem. Rev. 2017, 117, 12851-12892. [CrossRef] [PubMed]

3. Eom, T.; Yoo, W.; Kim, S.; Khan, A. Biologically activatable azobenzene polymers targeted at drug delivery and imaging applications. Biomaterials 2018, 185, 333-347. [CrossRef] [PubMed]

4. Stuart, M.A.C.; Huck, W.T.S.; Genzer, J.; Müller, M.; Ober, C.; Stamm, M.; Sukhorukov, G.B.; Szleifer, I.; Tsukruk, V.V.; Urban, M.; et al. Emerging applications of stimuli-responsive polymer materials. Nat. Mater. 2010, 9, 101-113. [CrossRef] [PubMed]

5. Rus, D.; Tolley, M.T. Design, fabrication and control of origami robots. Nature 2015, 521, 467-475. [CrossRef] [PubMed]

6. Lahikainen, M.; Zeng, H.; Priimagi, A. Reconfigurable photoactuator through synergistic use of photochemical and photothermal effects. Nat. Commun. 2018, 9, 4148. [CrossRef] [PubMed]

7. Yu, Y.; Nakano, M.; Ikeda, T. Directed bending of a polymer film by light. Nature 2003, 425, 145. [CrossRef] [PubMed]

8. Bandara, H.M.D.; Burdette, S.C. Photoisomerization in different classes of azobenzene. Chem. Soc. Rev. 2012, 41, 1809-1825. [CrossRef] [PubMed]

9. Aßhoff, S.J.; Lancia, F.; Iamsaard, S.; Matt, B.; Kudernac, T.; Fletcher, S.P.; Katsonis, N. High-power actuation from molecular photoswitches in enantiomerically paired soft springs. Angew. Chem. Int. Ed. 2017, 56, 3261-3265. [CrossRef]

10. Gelebart, A.H.; Jan Mulder, D.; Varga, M.; Konya, A.; Vantomme, G.; Meijer, E.W.; Selinger, R.L.B.; Broer, D.J. Making waves in a photoactive polymer film. Nature 2017, 546, 632-636. [CrossRef]

11. Wang, M.; Lin, B.P.; Yang, H. A plant tendril mimic soft actuator with phototunable bending and chiral twisting motion modes. Nat. Commun. 2016, 7, 13981. [CrossRef] [PubMed]

12. Takashima, Y.; Hatanaka, S.; Otsubo, M.; Nakahata, M.; Kakuta, T.; Hashidzume, A.; Yamaguchi, H.; Harada, A. Expansion-contraction of photoresponsive artificial muscle regulated by host-guest interactions. Nat. Commun. 2012, 3, 1270-1278. [CrossRef] [PubMed]

13. Iwaso, K.; Takashima, Y.; Harada, A. Fast response dry-type artificial molecular muscles with [c2] daisy chains. Nat. Chem. 2016, 8, 625-632. [CrossRef] [PubMed]

14. Garcia-Garibay, M.A. Molecular crystals on the move: From single-crystal-to-single-crystal photoreactions to molecular machinery. Angew. Chem. Int. Ed. 2007, 46, 8945-8947. [CrossRef] [PubMed]

15. Kobatake, S.; Takami, S.; Muto, H.; Ishikawa, T.; Irie, M. Rapid and reversible shape changes of molecular crystals on photoirradiation. Nature 2007, 446, 778-781. [CrossRef] [PubMed]

16. Irie, M.; Fukaminato, T.; Matsuda, K.; Kobatake, S. Photochromism of diarylethene molecules and crystals: Memories, switches, and actuators. Chem. Rev. 2014, 114, 12174-12277. [CrossRef]

17. Kim, T.; Zhu, L.; Al-Kaysi, R.O.; Bardeen, C.J. Organic photomechanical materials. Chem. Phys. Chem. 2014, 15, 400-414. [CrossRef]

18. Abendroth, J.M.; Bushuyev, O.S.; Weiss, P.S.; Barrett, C.J. Controlling motion at the nanoscale: Rise of the molecular machines. ACS Nano 2015, 9, 7746-7768. [CrossRef]

19. Naumov, P.; Chizhik, S.; Panda, M.K.; Nath, N.K.; Boldyreva, E. Mechanically responsive molecular crystals. Chem. Rev. 2015, 115, 12440-12490. [CrossRef]

20. Commins, P.; Desta, I.T.; Karothu, D.P.; Panda, M.K.; Naumov, P. Crystals on the move: Mechanical effects in dynamic solids. Chem. Commun. 2016, 52, 13941-13954. [CrossRef] 
21. Bushuyev, O.S.; Friščić, T.; Barrett, C.J. Photo-induced motion of azo dyes in organized media: From single and liquid crystals, to MOFs and machines. Cryst. Eng. Comm. 2016, 18, 7204-7211. [CrossRef]

22. Koshima, H.; Taniguchi, T.; Asahi, T. Chapter 3: Photomechanical and thermomechanical crystals. In Mechanically Responsive Materials for Soft Robotics; Koshima, H., Ed.; Wiley-VCH Verlag GmbH: Weinheim, Germany, 2019; pp. 57-82.

23. Brown, C.J. A refinement of the crystal structure of azobenzene. Acta Crystallogr. 1966, 21, 146-152. [CrossRef]

24. Mostad, A.; Rømming, C. A refinement of the crystal structure of cis-azobenzene. Acta Chem. Scand. 1971, 25, 3561-3568. [CrossRef]

25. Tsuda, M.; Kuratani, K. Isomerization of cis-azobenzene in the solid phase. Bull. Chem. Soc. Jpn. 1964, 37, 1284-1288. [CrossRef]

26. Koshima, H.; Ojima, N.; Uchimoto, H. Mechanical motion of azobenzene crystals upon photoirradiation. J. Am. Chem. Soc. 2009, 131, 6890-6891. [CrossRef] [PubMed]

27. Koshima, H.; Ojima, N. Photomechanical bending of 4-aminoazobenzene crystals. Dyes Pigment. 2012, 92, 798-801. [CrossRef]

28. Beharry, A.A.; Woolley, G.A. Azobenzene photoswitches for biomolecules. Chem. Soc. Rev. 2011, 40, 4422-4437. [CrossRef]

29. Bushuyev, O.S.; Singleton, T.A.; Barrett, C.J. Fast, reversible, and general photomechanical motion in single crystals of various Azo compounds using visible light. Adv. Mater. 2013, 25, 1796-1800. [CrossRef]

30. Timoshenko, S. Analysis of Bi-Metal Thermostats. J. Opt. Soc. Am. Rev. Sci. Instrum. 1925, 11, $233-255$. [CrossRef]

31. Kitagawa, D.; Iwaihara, C.; Nishi, H.; Kobatake, S. Quantitative evaluation of photoinduced bending speed of diarylethene crystals. Crystals 2015, 5, 551-561. [CrossRef]

32. Nath, N.K.; Pejov, L.; Nichols, S.M.; Hu, C.; Saleh, N.; Kahr, B.; Naumov, P. Model for photoinduced bending of slender molecular crystals. J. Am. Chem. Soc. 2014, 136, 2757-2766. [CrossRef] [PubMed]

33. Panda, M.K.; El Azhary, N.; Alzaabi, M.A.; Wahba, B.M.; Jacob, J.; Naumov, P. Photomechanical and photochromic behavior of a molecule containing multiple photoactive groups. Croat. Chem. Acta 2014, 87, 475-479. [CrossRef]

34. Zhu, L.; Al-Kaysi, R.O.; Bardeen, C.J. Reversible photoinduced twisting of molecular crystal microribbons. J. Am. Chem. Soc. 2011, 133, 12569-12575. [CrossRef] [PubMed]

35. Kitagawa, D.; Nishi, H.; Kobatake, S. Photoinduced twisting of a photochromic diarylethene crystal. Angezw. Chem. Int. Ed. 2013, 52, 9320-9322. [CrossRef] [PubMed]

36. Kim, T.; Zhu, L.; Mueller, L.J.; Bardeen, C.J. Mechanism of photoinduced bending and twisting in crystalline microneedles and microribbons composed of 9-methylanthracene. J. Am. Chem. Soc. 2014, 136, 6617-6625. [CrossRef] [PubMed]

37. Kitagawa, D.; Tsujioka, H.; Tong, F.; Dong, X.; Bardeen, C.J.; Kobatake, S. Control of photomechanical crystal twisting by illumination direction. J. Am. Chem. Soc. 2018, 140, 4208-4212. [CrossRef] [PubMed]

38. Taniguchi, T.; Fujisawa, J.; Shiro, M.; Koshima, H.; Asahi, T. Mechanical motion of chiral azobenzene crystals with twisting upon photoirradiation. Chem. Eur. J. 2016, 22, 7950-7958. [CrossRef] [PubMed]

39. Desiraju, G.R. Supramolecular synthons in crystal engineering-A new organic synthesis. Angew. Chem. Int. Ed. Engl. 1995, 34, 2311-2327. [CrossRef]

40. Saha, S.; Desiraju, G.R. Using structural modularity in cocrystals to engineer properties: Elasticity. Chem. Commun. 2016, 52, 7676-7679. [CrossRef]

41. Christopherson, J.C.; Topić, F.; Barrett, C.J.; Friščić, T. Halogen-bonded cocrystals as optical materials: Next-generation control over light-matter interactions. Cryst. Growth Des. 2018, 18, 1245-1259. [CrossRef]

42. Bushuyev, O.S.; Tomberg, A.; Friščić, T.; Barrett, C.J. Shaping crystals with light: Crystal-to-crystal isomerization and photomechanical effect in fluorinated azobenzenes. J. Am. Chem. Soc. 2013, 135, 12556-12559. [CrossRef] [PubMed]

43. Bushuyev, O.S.; Tomberg, A.; Vinden, J.R.; Moitessier, N.; Barrett, C.J.; Friščić, T. Azo ․ phenyl stacking: A persistent self-assembly motif guides the assembly of fluorinated cis-azobenzenes into photo-mechanical needle crystals. Chem. Commun. 2016, 52, 2103-2106. [CrossRef] [PubMed]

44. Bushuyev, O.S.; Corkery, T.C.; Barrett, C.J.; Friščić, T. Photo-mechanical azobenzene cocrystals and in situ X-ray diffraction monitoring of their optically-induced crystal-to-crystal isomerisation. Chem. Sci. 2014, 5, 3158-3164. [CrossRef] 
45. Cheng, S.C.; Chen, K.J.; Suzaki, Y.; Tsuchido, Y.; Kuo, T.S.; Osakada, K.; Horie, M. Reversible laser-induced bending of pseudorotaxane crystals. J. Am. Chem. Soc. 2018, 140, 90-93. [CrossRef] [PubMed]

46. Uchida, E.; Azumi, R.; Norikane, Y. Light-induced crawling of crystals on a glass surface. Nat. Commun. 2015, 6, 7310. [CrossRef] [PubMed]

47. Saito, K.; Ohnuma, M.; Norikane, Y. Negative phototactic behaviour of crystals on a glass surface. Chem. Commun. 2019, 55, 9303-9306. [CrossRef] [PubMed]

48. Norikane, Y.; Tanaka, S.; Uchida, E. Azobenzene crystals swim on water surface triggered by light. Cryst. Eng. Comm. 2016, 18, 7225-7228. [CrossRef]

49. Hao, Y.; Huang, S.; Guo, Y.; Hao, H.; Zhou, L.; Barrett, C.J.; Yu, H. Photoinduced multi-directional deformation of azobenzene molecular crystals. J. Mater. Chem. C 2019, 7, 503-508. [CrossRef]

50. Taniguchi, T.; Sugiyama, H.; Uekusa, H.; Shiro, M.; Asahi, T.; Koshima, H. Walking and rolling of crystals induced thermally by phase transition. Nat. Commun. 2018, 9, 538. [CrossRef]

51. Kitagawa, D.; Kawasaki, K.; Tanaka, R.; Kobatake, S. Mechanical behavior of molecular crystals induced by combination of photochromic reaction and reversible single-crystal-to-single-crystal phase transition. Chem. Mater. 2017, 29, 7524-7532. [CrossRef]

52. Taniguchi, T.; Sato, H.; Hagiwara, Y.; Asahi, T.; Koshima, H. Photo-triggered phase transition of a crystal. Commun. Chem. 2019, 2, 19. [CrossRef]

53. Lee, J.; Oh, S.; Pyo, J.; Kim, J.M.; Je, J.H. A light-driven supramolecular nanowire actuator. Nanoscale 2015, 7, 6457-6461. [CrossRef] [PubMed]

54. Momeni, F.; Hassani, N.S.M.M.; Liu, X.; Ni, J. A review of 4D printing. Mater. Des. 2017, 122, 42-79. [CrossRef]

55. Sydney Gladman, A.; Matsumoto, E.A.; Nuzzo, R.G.; Mahadevan, L.; Lewis, J.A. Biomimetic 4D printing. Nat. Mater. 2016, 15, 413-418. [CrossRef] [PubMed]

56. Morimoto, M.; Irie, M. A diarylethene cocrystal that converts light into mechanical work. J. Am. Chem. Soc. 2010, 132, 14172-14178. [CrossRef] [PubMed]

57. Koshima, H.; Matsuo, R.; Matsudomi, M.; Uemura, Y.; Shiro, M. Light-driven bending crystals of salicylidenephenylethylamines in enantiomeric and racemate forms. Cryst. Growth Des. 2013, 13, 4330-4337. [CrossRef]

58. Thorpe, S.K.; Li, Y.; Crompton, R.H.; Alexander, R.M. Stresses in human leg muscles in running and jumping determined by force plate analysis and from published magnetic resonance images. J. Exp. Biol. 1998, 201, 63-70.

59. Yu, Q.; Yang, X.; Chen, Y.; Yu, K.; Gao, J.; Liu, Z.; Cheng, P.; Zhang, Z.; Aguila, B.; Ma, S. Fabrication of light-triggered soft artificial muscles via a mixed-matrix membrane strategy. Angew. Chem. Int. Ed. 2018, 57, 10192-10196. [CrossRef]

(C) 2019 by the authors. Licensee MDPI, Basel, Switzerland. This article is an open access article distributed under the terms and conditions of the Creative Commons Attribution (CC BY) license (http://creativecommons.org/licenses/by/4.0/). 